\title{
Treatment and Survivorship Interventions to Prevent Poor Body Image Outcomes in Breast Cancer Survivors
}

\author{
Caroline Fiser' \\ Jessica S Crystal' \\ Sarah E Tevis ${ }^{2}$ \\ Susan Kesmodel ${ }^{1}$ \\ Kristin E Rojas' \\ 'Dewitt Daughtry Department of \\ Surgery, University of Miami/Miller \\ School of Medicine, Miami, FL, USA; \\ ${ }^{2}$ Department of Surgery, University of \\ Colorado, Anschutz Medical Campus, \\ Aurora, CO, USA
}

\begin{abstract}
Body image concerns often arise during and after treatment and are a major concern in up to $67 \%$ of breast cancer survivors. Negative changes in body image are a predictor of worse satisfaction with appearance and poor quality of life outcomes. Opportunities to mitigate the negative impact of cancer treatment on a patient's body image present during preoperative education or in the neoadjuvant setting, or during surgical management, adjuvant therapy delivery, and survivorship. The surgical management of breast cancer has evolved from breast amputations to procedures that provide improved cosmesis without compromising the oncologic outcome. The advent of the sentinel lymph node biopsy and lymphatic reconstruction techniques has led to decreased axillary morbidity. Modified radiation techniques and systemic therapies tailored to subtype limit unnecessary exposure to skin and systemic toxicities. Finally, incorporating prehabilitation and survivorship support optimizes the physical and psychosocial wellbeing of these patients. Setting expectations, treatment de-escalation when appropriate, morbidity risk reduction and improved screening and management of psychological sequelae during survivorship can decrease breast cancer treatment's negative impact on body image. The following review synthesizes interventions during preoperative planning, local and systemic treatment, and survivorship to prevent poor body image outcomes without compromising oncologic success.
\end{abstract}

Keywords: survivorship, oncoplastic surgery, lymphedema, body image, breast conservation, sentinel node biopsy

\section{Introduction}

Invasive female breast cancer incidence rates continue to increase by about $0.5 \%$ per year. Early diagnosis, increased incidence, and improved treatments combined with the aging population will lead to an estimated 281,550 new cases of invasive breast cancer diagnosed in women in 2021.,2 Changes in appearance and body image through treatment and in the adjuvant setting are a significant area of concern in breast cancer survivors, affecting anywhere from $31 \%-67 \%$ of these patients. ${ }^{3}$ Some of these changes are necessary for treatment and impact survival, while others can be mitigated with prevention strategies. While these concerns arising during and after treatment may be challenging to address, setting expectations, treatment de-escalation when appropriate, morbidity risk reduction, and improved screening and management of psychological and sexual sequelae during survivorship can decrease breast cancer treatment's negative impact on body image (Figure 1).
Correspondence: Kristin E Rojas

Dewitt Daughtry Department of Surgery,

University of Miami, 1295 NW 14th

Street, Miami, FL, 33I36, USA

Tel +I 305 243-0783

Email kristinrojasmd@gmail.com 
- Surgical de-escalation

- Oncoplastic procedures

- Axillary downstaging, lymphedema prevention techniques

- Improved radiation delivery

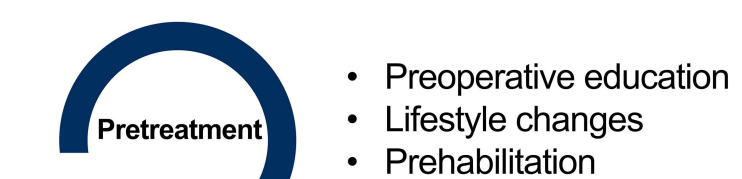

- Prehabilitation

- Screening for lymphedema

- Treating menopausal symptoms

- Addressing sexual health concerns

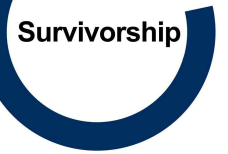

Figure I Interventions to decrease disruptions in body image through the breast cancer care continuum.

\section{Shared Decision-Making}

Preoperative education and expectation setting impacts patients' selection of surgical treatment that may include options of breast-conserving therapy (lumpectomy or oncoplastic lumpectomy), skin-sparing and non-skin-sparing mastectomy with and with or without reconstruction, and axillary staging. Before starting treatment, an open discussion on surgical options and their potential to change how patients may look and therefore their body image can assist with this complex decision-making. Often these conversations are an opportunity to dispel patients' misconceptions that more aggressive surgery is necessary to decrease the recurrence rate, which is usually not the case in early-stage breast cancer. ${ }^{4}$ Such discussions also improve objective surgical outcomes such as the rate of complications tied to poor patient satisfaction with appearance. The American Society of Breast Surgeons' multidisciplinary pain management consensus guidelines outline the importance of appropriate expectation-setting to improve postoperative pain control, further decreasing patient anxiety. They also recommend nutritional optimization, smoking cessation, activity instructions, and wound and drain care, impacting postoperative complication rates and cosmetic outcomes. ${ }^{5}$

\section{Local Therapy De-Escalation}

The contemporary management of localized breast cancer involves a multimodal approach that includes surgery and systemic therapy with or without adjuvant radiation. The past decade has produced a rapid evolution in the deescalation of treatment for patients with early-stage disease to include downstaging of breast and axillary surgery, tailored systemic therapies to ensure that only those who will benefit will receive them, and novel approaches to radiation delivery that include hypofractionation schedules and intensity-modulated radiation therapy (IMRT).

Surgery for breast cancer requires a delicate balance between oncologic control of the disease while preserving the aesthetic appearance of the breast. The surgical management of breast cancer has evolved from breast amputations during the Byzantine Empire, ${ }^{6}$ radical mastectomies pioneered by William Halsted in 1882, and eventually breast conservation, including oncoplastic procedures. The Halsted radical mastectomy includes resection of the chest wall skin, breast parenchyma, pectoralis major and minor muscles, and all axillary lymph nodes. ${ }^{7}$ In 1971, Fisher et al published the results of a landmark randomized trial which compared radical mastectomy to mastectomy with or without radiation therapy and demonstrated no improvement in survival with more extensive surgery. ${ }^{8}$

This finding led to the development of the simple mastectomy (removal of skin and complete breast parenchyma) with a tailored axillary approach. In the early 1990s, the skin-sparing mastectomy was introduced, where 
the mammary gland, pectoralis major fascia, and the nipple areolar complex are removed while preserving the skin envelope. ${ }^{9}$ Nipple-sparing mastectomies were first described as subcutaneous mastectomies in the early 1990s in the risk reduction setting. Of note, today's nipplesparing mastectomy does not purposely leave behind subcutaneous tissue as the original iteration and is considered an acceptable surgical strategy for carefully selected patients. In 2003, Gerber et al demonstrated their safety in patients with tumors distant from the nipple. ${ }^{10}$ The continued evolution of mastectomy techniques from Halsted's radical mastectomy to the nipple-sparing mastectomy has led to less morbidity and improved cosmesis without compromising oncologic outcomes. ${ }^{7}$ This is further highlighted in studies demonstrating improved psychosocial well-being in patients who receive nipplesparing mastectomy vs. total mastectomy. ${ }^{11}$ Nevertheless, despite preserving the native nipple-areolar complex, this approach can still result in decreased nipple sensation for women, impacting their arousal and sexual satisfaction. ${ }^{12}$

In addition to the contemporary mastectomy types, breast conservation (BCT), or lumpectomy with adjuvant radiation, is appropriate surgical management for women with early-stage disease. The NSABP B-06 trial demonstrated similar survival in women with early-stage breast cancer when comparing mastectomy to lumpectomy with or without radiation therapy. ${ }^{13}$ A more recent study reported that $\mathrm{BCT}$ results in superior survival outcomes compared with mastectomy with or without radiation after adjusting for tumor characteristics, treatment, demographics, comorbidity, and socioeconomic background. ${ }^{14}$ Breast conservation is an oncologically-safe procedure in appropriately-selected patients and is also more likely to result in superior satisfaction with appearance when compared to mastectomy with or without reconstruction. ${ }^{15}$

Even though lumpectomy removes less breast tissue, contour deformities may persist when $20 \%$ of breast volume has been removed or if the tumor resection is performed in the inferior, medial or retroareolar region. ${ }^{16}$ In appropriately selected patients, plastic surgery techniques can be combined with lumpectomy, allowing for more extensive resections and reconstruction of the defect to improve cosmesis. The basic principles of oncoplastic surgery, a term first used in 1993, involve aesthetic scar placement, reconstructing volume defects using either tissue remodeling or volume replacement using distant tissue, de-epithelialization, and donut mastopexy. ${ }^{17,18}$ Another tenet of oncoplastic surgery is to perform a symmetrizing procedure to the contralateral breast. When patients undergo BCT with mastopexy or a breast reduction technique, they can be offered simultaneous contralateral symmetrizing mastopexy without an increased risk of complication or delays to adjuvant therapy. ${ }^{19}$ Oncoplastics will remain an essential part of breast cancer treatment as multiple studies have shown improved objective cosmetic outcomes, patient-reported quality of life, and self-esteem compared to BCT alone. ${ }^{17}$

\section{Axillary Downstaging}

As the surgical technique of the mastectomy evolves, so too has the surgical management of the axilla. Determining axillary lymph node status in the preoperative setting provides prognostic information while guiding surgical, systemic, and radiation treatment decisions. Axillary lymph node dissection, which involves the removal of the lymph nodes in levels I and II of the axilla (lateral and posterior to the pectoralis minor muscle) carries significant risk of postoperative morbidity including decreased upper extremity mobility, seroma formation, paresthesia, and lymphedema. Of these, lymphedema has a significant impact on a patient's aesthetic and functional outcome. The prevalence of lymphedema in patients undergoing an axillary lymph node dissection is between 20 and $35 \%$ but varies depending on comorbid conditions and receipt of multimodal therapy. ${ }^{20}$ In patients presenting with clinically node-negative disease, sentinel lymph node biopsy has replaced axillary lymph node dissection as a staging procedure for the axilla. Sentinel node biopsy is a procedure that utilizes radiotracer, dye, or both in order to identify the first draining lymph nodes from the breast and the ones most likely to have cancer if the tumor had spread from the breast. The false negative rate of the procedure is $5-10 \% .^{21}$ The standard of care is to offer sentinel node biopsy in patients with clinically node-negative disease, which affords a significantly lower rate of lymphedema (5\%). ${ }^{20}$ Therefore, detecting breast cancer in the early stages, prior to metastasis to the axilla, translates to less longterm morbidity.

With the increased utilization of neoadjuvant therapy, the ability to "downstage" a patient's axilla from clinically node-positive to node-negative may allow for less extensive axillary surgery. Published results of the ACOSOG Z1071 and the SENTINA trials demonstrated that with appropriate staging and surgical techniques, the false negative rate could be decreased to an acceptably low percentage. $^{20,23}$ ACOSOG Z1071 was a single-arm study where all patients received neoadjuvant therapy, followed by an attempt at sentinel lymph node biopsy, followed by 
axillary lymph node dissection. The study found that the false-negative rate of sentinel node biopsy in women with clinically node-positive (cN1) disease who received neoadjuvant chemotherapy who had two or more sentinel lymph nodes examined was $12.6 \%$, which was greater than the predetermined threshold of $10 \%$. However, when the sentinel node biopsy included three or more sentinel lymph nodes and dual tracers were used for sentinel lymph node detection (both radionucleotide and blue dye), the false-negative rate fell to $10.8 \%$ and $7 \%$, respectively. ${ }^{22}$ Results from the SENTINA trial were similar with a false negative rate of less $10 \%$ for patients who had $\geq 3$ sentinel lymph nodes removed and a false negative rate of $8.6 \%$ with the use of dual tracers. ${ }^{23}$ These and other seminal studies led to the widespread adoption of sentinel node biopsy in patients with a good clinical response to neoadjuvant therapy in the axilla. Furthermore, utilizing less morbid axillary surgery in this setting has also led to the increased utilization of neoadjuvant systemic therapies, therefore increasing options for the surgical management of the breast due to smaller post-treatment tumor sizes.

\section{Radiation}

Radiation side effects include skin dermatitis, skin retraction, scarring, capsular contracture, and decreased arm range of motion, all of which can negatively impact a patient's posttreatment body image. One way to reduce the incidence of these sequelae is to limit radiation in patients without a a survival benefit, specifically in older women. The CALGB 9343 trial showed that omission of radiotherapy in women $\geq 70$ years old with estrogen receptor-positive early-stage breast cancer who undergo lumpectomy and receive five years of endocrine therapy was not associated with worse overall survival. The study found no difference in overall or distant disease-free survival between those who did and did not receive adjuvant radiation. ${ }^{24}$ Similar results were demonstrated in the PRIME II clinical trial, which included women $\geq 65$ years old with early-stage breast cancer who underwent lumpectomy and received endocrine therapy and did not show a difference in regional recurrence, distant metastases, contralateral breast cancer rate or overall survival. ${ }^{25}$

Some patients undoubtedly benefit from adjuvant radiation, including those with histologically aggressive subtypes or node-positive disease. For patients requiring adjuvant radiation, body image-impacting adverse outcomes can be minimized through the modification of radiation delivery. For example, intensity-modulated radiation therapy (IMRT) utilizes computer-controlled linear accelerators to control the beam's intensity in multiple small volumes. IMRT allows higher doses to be focused on the tumor while limiting the radiation dose to adjacent normal tissue. Several studies have demonstrated improved cosmetic outcomes with the use of IMRT compared to standard radiotherapy. ${ }^{26-29}$ While not currently considered the standard of care for adjuvant breast cancer treatment, the use of proton radiotherapy instead of conventional photons may offer similar benefits to IMRT such as higher beam conformality, full coverage of the target while minimizing dose to surrounding tissues. Prospective studies are underway to determine whether proton therapy results in decreased skin toxicities compared to conventional photons. ${ }^{30}$

\section{Systemic Therapy}

Neoadjuvant or adjuvant systemic therapy is a key element in the multimodal approach to treating invasive breast cancer. Genomic profiling of tumors allows for the selection of patients most likely to benefit from cytotoxic chemotherapy whose adverse effects include but are not limited to neuropathy, alopecia, cardiotoxicity, cytopenias, and induced menopause. The TAILORx and RxPonder trials randomized early-stage patients with estrogen receptor positive, Her2-negative (ER +/Her2-) invasive breast cancer to receive adjuvant endocrine therapy alone or chemotherapy and endocrine therapy based on Oncotype DX Recurrence Score. ${ }^{31,32}$ The TAILORx trial found no benefit to chemotherapy in women with nodenegative ER+/Her2- and a Recurrence Score (RS) of 25 or less. ${ }^{31}$ The RxPonder trial addressed a similar question in women with node-positive ER+/Her2- disease (1-3 nodes positive) and found that postmenopausal women with a RS of 25 or less can forgo chemotherapy regardless of clinicalpathological parameters. In premenopausal women, however, with similar RS there was some benefit from chemotherapy. ${ }^{32}$ More work is currently underway to determine if certain groups within this premenopausal population can omit cytotoxic chemotherapy without an adverse oncologic impact. The utilization of genomic profiling has significantly impacted the treatment of invasive breast cancer, with approximately $70 \%$ of patients with early-stage ER+/Her2- breast cancer able to safely avoid chemotherapy. ${ }^{31}$

Most chemotherapy regimens, until recently, included either an anthracycline or a taxane agent. Both drugs are known to cause temporary or rarely permanent alopecia, which can negatively impact body image. The incorporation of scalp cooling devices during chemotherapy receipt now offers patients an option for limiting hair loss during treatment. Recent work found successful hair preservation in 
approximately $50 \%$ of women with scalp cooling compared to $0 \%$ of women in the control group $(\mathrm{p}=0.006) .{ }^{33}$ Unfortunately, the commercially available devices are not always covered by insurance and sometimes result in an out-of-pocket expense for patients.

\section{Lymphedema Risk Mitigation}

Lymphedema is the localized swelling caused by an abnormal accumulation of protein-rich lymphatic fluid in the subcutaneous tissues. One in five of the current 3.5 million breast cancer survivors in the United States will be diagnosed with lymphedema during their lifetime. ${ }^{34}$ However, with the adoption of sentinel lymph node biopsy instead of a complete axillary lymph node dissection in clinically node-negative breast cancer patients, the median number of removed nodes and associated axillary lymphatics decreases, lowering the risk of lymphedema from $14 \%$ to $8 \%$. $^{35}$

If an axillary dissection is required either because of bulky nodal disease, incomplete response to neoadjuvant chemotherapy, or to avoid post-mastectomy radiation, a proactive approach to lymphedema prevention may be incorporated into the surgical procedure. One method to reduce lymphedema is to preserve lymphatics draining from the arm through axillary reverse mapping (ARM). ARM was developed in the 1960s and involves injecting blue dye into the subdermal space of the upper medial arm before the start of the axillary lymphadenectomy. The blue dye is taken up by the lymphatics in the axilla which can be identified and carefully preserved during the axillary surgery. ${ }^{36}$

In 1969, the first lymphaticovenous bypass was described to treat patients with chronic lymphedema. The principles of this procedure are to anastomose lymphatic channels to nearby veins to bypass the diseased or damaged lymphatic channels. The contemporary LYMPHA technique (Lymphatic Microsurgical Preventive Healing Approach) can be utilized during the axillary lymphadenectomy with a surgical microscope to anastomose the blue lymphatics to a vein. Recent work found that with the utilization of this technique, the lymphedema rate is as low as $4 \%{ }^{37}$ Another option to treat longstanding lymphedema is Vascularized Lymph Node Transfer (VLNT) utilizing microsurgical techniques to transplant functional lymph nodes into the recipient bed of an extremity affected by lymphedema in order to reestablish lymphatic function and flow to the affected extremity. $^{38}$

\section{Preventing and Managing Weight Gain During Treatment}

Weight gain is a common problem for women diagnosed with breast cancer and occurs from the time of diagnosis and continues up to three years post-diagnosis. Fatigue, decreased lean muscle mass, metabolic rate changes, and the induction of menopause in premenopausal women all contribute to weight gain during treatment. ${ }^{39,40}$ In a cross-sectional survey of patients at least one year from surgery, women who were obese were more dissatisfied with their appearance than normal-weight women ( 13.0 vs $4.1 \%, \mathrm{p}=0.01)$. Of note, this adverse impact on body image includes women who receive mastectomy with reconstruction. ${ }^{41}$

Outside of the psychosocial impact, addressing weight management before the initiation of treatment may also impact oncologic outcomes. Exercising during breast cancer treatment not only helps with weight loss, but also decreases the risk of recurrence, anxiety and fatigue, and improves overall quality of life. This is beneficial for patients with obesity, which has been shown to lead to an increased risk of breast cancer recurrence. ${ }^{42}$ A systematic review looking at prevention interventions to prevent weight gain in women undergoing chemotherapy for breast cancer found that the most successful interventions promoted physical activity multiple times per week and face-to-face dietary counseling. With these interventions, women were able to prevent weight gain and even lose weight during treatment. ${ }^{39}$

\section{Prehabilitation}

Improving patients' physical preparedness for treatment is an intervention that can improve treatment outcomes, decrease complication rates, and improve postoperative body image and satisfaction. Several studies have shown that physically fit surgical candidates have lower perioperative complication rates and faster return to baseline function. "Prehabilitation" is defined as the "process on the continuum of care that occurs between the time of cancer diagnosis and the beginning of acute treatment, includes physical and psychological assessments that establish a baseline functional level, identifies impairments, and provides targeted interventions that improve a patient's health to reduce the incidence and the severity of current and future impairments. ${ }^{43}$ The essential components of a prehab program include general conditioning exercise, targeted exercise, nutritional interventions, psychological well-being, and smoking cessation. ${ }^{44}$ In terms of general conditioning exercises, muscle groups are 
targeted via resistance, flexibility, or strength training. Prescribing "prehabilitation" before initiating systemic therapies can help manage treatment-associated weight gain while improving both objective and patient-reported outcomes. Patients with better cardiovascular status prior to initiation of chemotherapy may also have decreased morbidity and less cardiotoxicity. ${ }^{45}$

Prehabilitation prior to surgery can also impact surgical outcomes. An expert panel recently published guidelines illustrating the role of nutrition, exercise, stress, and smoking and their effects on perioperative outcomes. ${ }^{46}$ Recent studies have shown that prehabilitation leads to a reduction of postoperative complications and a faster recovery after surgery. ${ }^{47}$ Studies are ongoing in the autologous breast reconstruction setting to determine whether "prehab" targeting of the shoulders, arms, and abdomen to increase range of motion and strength improves postoperative reconstruction outcomes. ${ }^{48}$ Prehabilitation is an evolving therapy that seems to be beneficial to breast cancer patients and can help with their overall health, strength, mobility along with an improved sense of self.

\section{Addressing Menopausal and Sexual Sequelae of Treatment}

Women's cancer treatment may lead to changes in sexual health including induced menopause, and adversely impact their perception of physical attractiveness. ${ }^{49}$ Patients receiving either chemotherapy and/or estrogen-blocking medications may experience early menopause or a worsening of menopausal symptoms including hot flashes, metabolic disruptions, mood changes, musculoskeletal pain, and sexual dysfunction. ${ }^{50}$ Premenopausal patients receiving chemotherapy are often prescribed ovarian suppression to "quiet" the ovaries during chemotherapy, protecting them from cytotoxicity and improving ovarian function post-treatment. ${ }^{51,52}$ Similarly, the gonadotoxicity induced through exposure to cytotoxic chemotherapy without the use of gonadotropinaltering medications as ovarian suppression may produce similar symptoms.

After surgery with or without radiation or chemotherapy, patients with estrogen receptor-positive breast cancer are usually recommended 5-10 years of adjuvant endocrine therapy (also known as estrogen blockade) to decrease the risk of local and systemic recurrence. ${ }^{52}$ Aromatase inhibitors (AIs) are recommended in this setting for postmenopausal or premenopausal patients on ovarian suppression. Their effectiveness depends on the systemic suppression of aromatase, leading to very low levels of circulating estrogens and oftentimes severe symptoms related to this suppression. Selective estrogen receptor modulators can be prescribed for either preor postmenopausal patients and produce similar symptoms through a different mechanism acting as an antagonist to the intranuclear estrogen receptor.

Preparing patients for these changes and pre-emptively offering strategies for symptom mitigation can improve patient quality of life and treatment adherence. Interventions to decrease hot flashes include exercise, avoiding triggers, and even acupuncture. Both venlafaxine and oxybutynin have been shown to decrease hot flash frequency and duration in prospective studies. ${ }^{53}$

Education regarding sexual sequelae of treatment such as vaginal dryness and atrophy symptoms, now known as genitourinary syndrome of menopause (GSM), fosters open communication during treatment and empowers patients to manage issues as they arise. Vaginal moisturizers with hyaluronic acid should be encouraged on evenings where sexual activity is not planned, and gimmick-free silicone-based lubricants for sexual activity can decrease intercourse-related pain.

While adjuvant endocrine therapy decreases the risk of recurrence by approximately $40 \%$, patient adherence to therapy relies on screening and treating symptoms. ${ }^{54}$ After three years of estrogen blockade with an aromatase inhibitor, the proportion of patients continuing to take the medication ranges from $50 \%-68 \%{ }^{55}$ Reasons for cessation of therapy in these studies include arthralgias, myalgias, hot flashes and fatigue. ${ }^{56}$ The American Cancer Society and American Society of Clinical Oncology have released a Breast Cancer Survivorship Care Guideline document in which five key areas of breast cancer survivorship are discussed. ${ }^{57}$ These five areas are surveillance for breast cancer recurrence, screening for second primary cancers, assessment and management of physical and psychosocial long-term effects of breast cancer and treatment, and promotion of health and coordination of care. Through increased awareness of the side effects of breast cancer treatment, from psychosocial issues to physical limitations, clinicians can address these issues and provide individualized support to patients.

\section{Psychological Interventions During Treatment}

Psychological interventions to address body image changes in breast cancer patients include cognitivebehavioral, educational, interpersonal, and psychosocial approaches with the aim of decreasing psychological 
distress, relaxation methods, and to modify the perception of one's body. ${ }^{58,59}$ As noted above, interventions including physical activity have the ability to decrease appearanceimpacting complications of surgery, but can also be combined with psychological support to promote personal strength as well as improvements in cognition. ${ }^{60}$ Cognitive Behavioral Therapy (CBT) is a time-limited and goal-oriented approach that focuses on changing patterns of thinking or behavior and has been shown to be helpful in addressing body image disturbances associated with cancer diagnosis and treatment. ${ }^{61}$ One-on-one psychotherapy, structured groups, and couple interventions were also found to improve body image scores and relationship satisfaction. ${ }^{61,62}$

\section{Discussion}

In 2022, nearly 4 million women will be living with a diagnosis of breast cancer. ${ }^{62}$ The impact of breast cancer on the well-being of patients is far-reaching and, unfortunately long-lasting, with body image a significant concern for many women. Furthermore, anxiety, depression, neurocognitive dysfunction, sexual dysfunction, and suicide are more common in breast cancer survivors when compared to non-cancer groups. ${ }^{63}$ Advancements in preoperative counseling, local therapy, tailored systemic treatments, and survivorship support can reduce the impact of treatment on a patient's perception of self. From a surgical standpoint, aesthetic outcomes can be optimized while preserving the oncologic principles of locoregional control and staging. Novel techniques such as oncoplastic and lymphatic-venous bypass procedures can decrease postoperative morbidities from chronic pain to lymphedema. Adjuvant therapy morbidity impacting body image such as skin changes from radiation are reduced through careful patient selection and improved delivery techniques. From a systemic therapies perspective, tumor-specific genomic profiling limits potential toxicities to only those known to benefit, while ancillary therapies such as scalp cooling devices can limit dramatic appearance changes associated with chemotherapy. Finally, the incorporation of prehabilitation and survivorship support, including screening for menopausal symptoms and sexual sequelae, can improve patient adherence to treatment and overall quality of life, optimizing the physical and psychologic well-being over their lifetime.

Of note, in this review, we focus on the different components of mitigating poor body image outcomes in the average female breast cancer patient, who is a median age of 62 years old. Consequently, some of these principles may not apply to all patients, such as younger patients in their $20 \mathrm{~s}-30 \mathrm{~s}$, a population that appears to be increasing over time. Likewise, given the low incidence of breast cancer in men, inferences are made based upon studies performed on women due to the exclusion of male patients from many large prospective trials. Ongoing inclusive trials will shed light on the impact of treatment on body image and appearance satisfaction in these unique patient populations. Nevertheless, increasing awareness regarding the potential sequelae of treatment for all patient populations will undoubtedly lead to improved screening measures and more options for treatment.

\section{Conclusion}

Women with breast cancer experience treatment-induced changes in appearance affecting their relationship with their body in some way. While some changes are necessary for treatment and improve survival, others can be mitigated with prevention strategies. Before starting treatment, an open discussion on surgical options and shared decision-making helps prepare patients for the changes they may experience. This conversation is an opportunity to dispel patients' misconceptions and clarify that less aggressive surgery may be feasible. Treatment deescalation when appropriate and interventions to reduce morbidity risk include utilization of neoadjuvant therapy, consideration of breast conservation, sentinel node biopsy and surgical interventions to prevent lymphedema can decrease abrupt or chronic physical alterations impacting patients' body image. Lastly, improved screening and management of psychological sequelae during survivorship, including addressing menopausal and sexual health concerns can decrease breast cancer treatment's negative impact on physical and psychological well-being and overall sense of bodily self.

\section{Acknowledgments}

The authors would like to acknowledge Jorge Madden who contributed to the design of Figure 1.

\section{Disclosure}

The authors report no conflicts of interest in this work.

\section{References}

1. SEER*Explorer: an interactive website for SEER cancer statistics. Surveillance Research Program, National Cancer Institute. Available from: https://seer.cancer.gov/explorer/. Accessed September 15, 2021. 
2. American Cancer Society. Cancer Facts \& Figures 2021. Atlanta: American Cancer Society; 2021. Available from: https://www.can cer.org/content/dam/cancer-org/research/cancer-facts-and-statistics /annual-cancer-facts-and-figures/2021/cancer-facts-and-figures-2021. pdf. Accessed September 15, 2021.

3. Falk Dahl CA, Reinertsen KV, Nesvold IL, Fosså SD, Dahl AA. A study of body image in long-term breast cancer survivors. Cancer. 2010;116(15):3549-3557. doi:10.1002/cncr.25251

4. Boniface J, Szulkin R, Johansson A. Survival After Breast Conservation vs Mastectomy Adjusted for Comorbidity and Socioeconomic Status: a Swedish National 6-Year Follow-up of 48986 Women. JAMA Surg. 2021;156(7):628-637. doi:10.1001/ jamasurg.2021.1438

5. Brenin DR, Dietz JR, Baima J, et al. Pain Management in Breast Surgery: recommendations of a Multidisciplinary Expert Panel-The American Society of Breast Surgeons. Ann Surg Oncol. 2020;27 (12):4588-4602. doi:10.1245/s10434-020-08892-x

6. Faguet GB. A brief history of cancer: age-old milestones underlying our current knowledge database. Int $J$ Cancer. 2015;136 (9):2022-2036. doi:10.1002/ijc.29134

7. Plesca M, Bordea C, El Houcheimi B, Ichim E, Blidaru A. Evolution of radical mastectomy for breast cancer. J Med Life. 2016;9(2):183-186.

8. Fisher B. United States trials of conservative surgery. World J Surg. 1977;1:327-330. doi:10.1007/BF01556850

9. Carlson GW. Skin sparing mastectomy: anatomic and technical considerations. Am Surg. 1996;62:151-155.

10. Gerber B, Krause A, Reimer T, et al. Skin-sparing mastectomy with conservation of the nipple-areola complex and autologous reconstruction is an oncologically safe procedure. Ann Surg. 2003;238 (1):120-127. doi:10.1097/01.SLA.0000077922.38307.cd

11. Romanoff A, Zabor EC, Stempel M, Sacchini V, Pusic A, Morrow M. A comparison of patient-reported outcomes after nipple-sparing mastectomy and conventional mastectomy with reconstruction. Ann Surg Oncol. 2018;25(10):2909-2916. doi:10.1245/s10434-018-6585-4

12. Bober SL, Varela VS. Sexuality in adult cancer survivors: challenges and intervention. J Clin Oncol. 2012;30:3712-3719. doi:10.1200/ JCO.2012.41.7915

13. Fisher B, Anderson S, Bryant J, et al. Twenty-year follow-up of a randomized trial comparing total mastectomy, lumpectomy, and lumpectomy plus irradiation for the treatment of invasive breast cancer. $N$ Engl J Med. 2002;347(16):1233-1241. doi:10.1056/ NEJMoa022152

14. De Boniface J, Szulkin R, Johansson ALV. Survival After Breast Conservation vs Mastectomy Adjusted for Comorbidity and Socioeconomic Status: a Swedish National 6-Year Follow-up of 48 986 Women. JAMA Surg. 2021;156(7):628-637.

15. Lagendijk M, van Egdom LSE, van Veen FEE, et al. Patient-reported outcome measures may add value in breast cancer surgery. Ann Surg Oncol. 2018;25(12):3563-3571. doi:10.1245/s10434-018-6729-6

16. Kuerer HM. Breast Surgical Oncology. 1st ed. New York: McGrawHill; 2010.

17. Kopkash K, Clark P. Basic Oncoplastic Surgery for Breast Conservation: tips and Techniques. Ann Surg Oncol. 2018;25 (10):2823-2828. doi:10.1245/s10434-018-6604-5

18. Gainer SM, Lucci A. Oncoplastics: techniques for reconstruction of partial breast defects based on tumor location. J Surg Oncol. 2011;103(4):341-347. doi:10.1002/jso.21672

19. Deigni OA, Baumann DP, Adamson KA, et al. Immediate Contralateral Mastopexy/Breast Reduction for Symmetry Can Be Performed Safely in Oncoplastic Breast-Conserving Surgery. Plast Reconstr Surg. 2020;145 (5):1134-1142. doi:10.1097/PRS.0000000000006722

20. Nguyen TT, Hoskin TL, Habermann EB, Cheville AL, Boughey JC. Breast cancer-related lymphedema risk is related to multidisciplinary treatment and not surgery alone: results from a large cohort study. Ann Surg Oncol. 2017;24(10):2972-2980. doi:10.1245/s10434-0175960-x
21. Kim T, Giuliano AE, Lyman GH. Lymphatic mapping and sentinel lymph node biopsy in early-stage breast carcinoma: a meta-analysis. Cancer. 2006;106:4-16. doi:10.1002/cncr.21568

22. Boughey JC, Suman VJ, Mittendorf EA, et al. Sentinel lymph node surgery after neoadjuvant chemotherapy in patients with node-positive breast cancer: the ACOSOG Z1071 (Alliance) clinical trial. JAMA. 2013;310(14):1455-1461. doi:10.1001/jama.2013.278932

23. Kuehn T, Bauerfeind I, Fehm T, et al. Sentinel-lymph-node biopsy in patients with breast cancer before and after neoadjuvant chemotherapy (SENTINA): a prospective, multicentre cohort study. Lancet Oncol. 2013;14(7):609-618. doi:10.1016/S1470-2045(13)70166-9

24. Hughes KS, Schnaper LA, Bellon JR, et al. Lumpectomy plus tamoxifen with or without irradiation in women age 70 years or older with early breast cancer: long-term follow-up of CALGB 9343. J Clin Oncol. 2013;31(19):2382-2387. doi:10.1200/JCO.2012.45.2615

25. Kunkler IH, Williams LJ, Jack WJ, Cameron DA, Dixon JM. PRIME II investigators. Breast-conserving surgery with or without irradiation in women aged 65 years or older with early breast cancer (PRIME II): a randomised controlled trial. Lancet Oncol. 2015;16(3):266-273. doi:10.1016/S1470-2045(14)71221-5

26. Barnett GC, Wilkinson JS, Moody AM, et al. The Cambridge Breast Intensity-modulated Radiotherapy Trial: patient- and treatment-related factors that influence late toxicity. Clin Oncol. 2011;23:662-673. doi:10.1016/j.clon.2011.04.011

27. Mukesh MB, Barnett G, Cumming J, et al. Association of breast tumour bed seroma with postoperative complications and late normal tissue toxicity: results from the Cambridge Breast IMRT trial. Eur J Surg Oncol. 2012;38:918-924. doi:10.1016/j.ejso.2012.05.008

28. Livi L, Meattini I, Marrazzo L, et al. Accelerated partial breast irradiation using intensity-modulated radiotherapy versus whole breast irradiation: 5-year survival analysis of a Phase 3 randomised controlled trial. Eur J Cancer. 2015;51:451-463. doi:10.1016/j. ejca.2014.12.013

29. Meattini I, Saieva C, Marrazzo L, et al. Accelerated partial breast irradiation using intensity-modulated radiotherapy technique compared to whole breast irradiation for patients aged 70 years or older: subgroup analysis from a randomized phase 3 trial. Breast Cancer Res Treat. 2015;153:539-547. doi:10.1007/s10549-015-3565-2

30. Corbin K, Mutter R. Proton therapy for breast cancer: progress \& pitfalls. Breast Cancer Management. 2018;7(1):1758-1931. doi:10.2217/bmt-2018-0001

31. Sparano JA, Gray RJ, Makower DF, et al. Adjuvant Chemotherapy Guided by a 21-Gene Expression Assay in Breast Cancer. $N$ Engl $J$ Med. 2018;379(2):111. doi:10.1056/NEJMoa1804710

32. Kalinsky K, Barlow WE, Meric-Bernstam F, et al. First results from a Phase III randomized clinical trial of standard adjuvant endocrine therapy \pm chemotherapy in patients with $1-3$ positive nodes, hormone receptor-positive and HER2-negative breast cancer with recurrence score<25: SWOG S1007 (RxPonder). Cancer Res. 2020;81S:SABCS \#GS3.

33. Nangia J, Wang T, Osborne C, et al. Effect of a Scalp Cooling Device on Alopecia in Women Undergoing Chemotherapy for Breast Cancer: the SCALP Randomized Clinical Trial. JAMA. 2017;317(6):596-605. doi:10.1001/jama.2016.20939

34. Miller KD, Sigel RL, Lin CC, et al. Cancer treatment and survivorship statistics, 2016. CA Cancer J Clin. 2016;66:271-289. doi:10.3322/caac. 21349

35. Ashikaga T, Krag DN, Land SR, et al. Morbidity results from the NSABP B-32 trial comparing sentinel lymph node dissection versus axillary dissection. J Surg Oncol. 2010;102(2):111-118. doi:10.1002/jso.21535

36. Johnson AR, Singhal D. Immediate lymphatic reconstruction. J Surg Oncol. 2018;118(5):750-757. doi:10.1002/jso.25177

37. Boccardo F, Casabona F, De Cian F, et al. Lymphedema microsurgical preventive healing approach: a new technique for primary prevention of arm lymphedema after mastectomy. Ann Surg Oncol. 2009;16(3):703-708. doi:10.1245/s10434-008-0270-y 
38. Schaverien MV, Badash I, Patel KM, Selber JC, Cheng MH. Vascularized Lymph Node Transfer for Lymphedema. Semin Plast Surg. 2018;32(1):28-35. doi:10.1055/s-0038-1632401

39. Thomson ZO, Reeves MM. Can weight gain be prevented in women receiving treatment for breast cancer? A systematic review of intervention studies. Obes Rev. 2017;18(11):1364-1373. doi:10.1111/ obr. 12591

40. Rojas KE, Matthews N, Raker C, et al. Body mass index (BMI), postoperative appearance satisfaction, and sexual function in breast cancer survivorship. J Cancer Surviv. 2018;12:127-133. doi:10.1007/ s11764-017-0651-y

41. Ewertz M, Jensen MB, Gunnarsdottir KA, et al. Effect of obesity on prognosis after early-stage breast cancer. $J$ Clin Oncol. 2011;29:25-31. doi:10.1200/JCO.2010.29.7614

42. Campbell KL, Winters-Stone KM, Wiskemann J, et al. Exercise Guidelines for Cancer Survivors: consensus Statement from International Multidisciplinary Roundtable. Med Sci Sports Exerc. 2019;51(11):2375-2390. doi:10.1249/MSS.0000000000002116

43. Silver JK, Baima J. Cancer prehabilitation: an opportunity to decrease treatment-related morbidity, increase cancer treatment options, and improve physical and psychological health outcomes. Am J Phys Med Rehabil. 2013;92(8):715-727. doi:10.1097/ PHM.0b013e31829b4afe

44. Santa Mina D, Brahmbhatt P, Lopez C, et al. The Case for Prehabilitation Prior to Breast Cancer Treatment. PM \& R: The Journal of Injury, Function, and Rehabilitation. 2017;9(9S2):S305S316. doi:10.1016/j.pmrj.2017.08.402

45. Wonders KY, Hydock DS, Schneider CM, Hayward R. Acute exercise protects against doxorubicin cardiotoxicity. Integr Cancer Ther. 2008;7(3):147-154. doi:10.1177/1534735408322848

46. Carli F, Silver JK, Feldman LS, et al. Surgical Prehabilitation in Patients with Cancer: state-of-the-Science and Recommendations for Future Research from a Panel of Subject Matter Experts. Phys Med Rehabil Clin N Am. 2017;28(1):49-64. doi:10.1016/j. pmr.2016.09.002

47. Molenaar CJL, Papen-Botterhuis NE, Herrle F, Slooter GD. Prehabilitation, making patients fit for surgery - a new frontier in perioperative care. Innov Surg Sci. 2019;4(4):132-138. doi:10.1515/ iss-2019-0017

48. Yang A, Sokolof J, Gulati A. The effect of preoperative exercise on upper extremity recovery following breast cancer surgery: a systematic review. Int $J$ Rehabil Res. 2018;41(3):189-196. doi:10.1097/MRR.0000000000000288

49. Sebri V, Durosini I, Triberti S, Pravettoni G. The Efficacy of Psychological Intervention on Body Image in Breast Cancer Patients and Survivors: a Systematic-Review and Meta-Analysis. Front Psychol. 2021;12:611954. doi:10.3389/fpsyg.2021.611954

50. Francis PA, Pagani O, Fleming GF, et al. Tailoring adjuvant endocrine therapy for premenopausal breast cancer. $N$ Engl J Med. 2018;379(2):122-137. doi:10.1056/NEJMoa1803164
51. Lambertini M, Moore HCF, Leonard RCF, et al. GonadotropinReleasing Hormone Agonists During Chemotherapy for Preservation of Ovarian Function and Fertility in Premenopausal Patients With Early Breast Cancer: a Systematic Review and Meta-Analysis of Individual Patient-Level Data. J Clin Oncol. 2018;36(19):1981-1990. doi:10.1200/JCO.2018.78.0858

52. Goss PE, Ingle JN, Pritchard KI, et al. Extending Aromatase-Inhibitor Adjuvant Therapy to 10 Years. $N$ Engl J Med. 2016;375(3):209-219. doi:10.1056/NEJMoa1604700

53. Leon-Ferre RA, Novotny PJ, Wolfe EG, et al. Oxybutynin vs Placebo for Hot Flashes in Women With or Without Breast Cancer: a Randomized, Double-Blind Clinical Trial (ACCRU SC-1603). JNCI Cancer Spectr. 2019;4(1):pkz088. doi:10.1093/jncics/pkz088

54. Kadakia KC, Henry NL. Adjuvant endocrine therapy in premenopausal women with breast cancer. Clin Adv Hematol Oncol. 2015;13 (10):663-672.

55. Partridge AH, LaFountain A, Mayer E, Taylor BS, Winer E, AsnisAlibozek A. Adherence to initial adjuvant anastrozole therapy among women with early-stage breast cancer. J Clin Oncol. 2008;26 (4):556-562. doi:10.1200/JCO.2007.11.5451

56. Hadji P. Improving compliance and persistence to adjuvant tamoxifen and aromatase inhibitor therapy. Crit Rev Oncol Hematol. 2010;73 (2):156-166. doi:10.1016/j.critrevonc.2009.02.001

57. Runowicz CD, Leach CR, Henry NL, et al. American Cancer Society/American Society of Clinical Oncology Breast Cancer Survivorship Care Guideline. CA Cancer J Clin. 2016;66(1):43-73.

58. Blanco C, Markowitz JC, Hershman DL, et al. Study of Interpersonal Psychotherapy for Depressed Women with Breast Cancer. Am J Psychother. 2014;68(4):489-495.

59. Sebri V, Savioni L, Triberti S, Mazzocco K, Pravettoni G. How to Train Your Health: sports as a Resource to Improve Cognitive Abilities in Cancer Patients. Front Psychol. 2019;10:2096. doi:10.3389/fpsyg.2019.02096

60. Fingeret MC, Teo I, Epner DE. Managing body image difficulties of adult cancer patients: lessons from available research. Cancer. 2014;120(5):633-641. doi:10.1002/cncr.28469

61. Kalaitzi C, Papadopoulos VP, Michas K, Vlasis K, Skandalakis P, Filippou D. Combined brief psychosexual intervention after mastectomy: effects on sexuality, body image, and psychological well-being. J Surg Oncol. 2007;96(3):235-240. doi:10.1002/jso.20811

62. de Moor JS, Mariotto AB, Parry C, et al. Cancer survivors in the United States: prevalence across the survivorship trajectory and implications for care. Cancer Epidemiol Biomarkers Prev. 2013;22 (4):561-570. doi:10.1158/1055-9965.EPI-12-1356

63. Carreira H, Williams R, Müller M, Harewood R, Stanway S, Bhaskaran K. Associations Between Breast Cancer Survivorship and Adverse Mental Health Outcomes: a Systematic Review. J Natl Cancer Inst. 2018;110(12):1311-1327. doi:10.1093/jnci/djy177

\section{Publish your work in this journal}

Breast Cancer - Targets and Therapy is an international, peer-reviewed open access journal focusing on breast cancer research, identification of therapeutic targets and the optimal use of preventative and integrated treatment interventions to achieve improved outcomes, enhanced survival and quality of life for the cancer patient.
The manuscript management system is completely online and includes a very quick and fair peer-review system, which is all easy to use. Visit http://www.dovepress.com/testimonials.php to read real quotes from published authors. 